\title{
IMPORTANCIA DE LA ENFERMERÍA BASADA EN EVIDENCIA
}

\author{
Importance Of Evidence-Based Nursing
}

\section{Dra. Isabel Natividad Urure Velazco ${ }^{1}$}

\begin{abstract}
1 Profesor principal de la Facultad de Enfermería, Universidad Nacional San Luis Gonzaga de Ica, Perú. Editor en Jefe de la REVAN (Revista Enfermería a la Vanguardia) Ica, Perú.
\end{abstract}

Al hablar de enfermería basada en evidencias, hoy en día significa un avance científico de la profesión y es concretamente referirnos a la utilización en forma crítica de los resultados de investigación científica y establecer mejoras en la calidad de los cuidados de enfermería en la práctica asistencial. Para lo cual se debe hacer una revisión bibliográfica minuciosa de la calidad de los resultados de investigación. Siendo de importancia la enfermería basada en evidencia porque va a permitir utilizar resultados obtenidos de una investigación científica, rescatar esta información valiosa, para luego aplicarlos a través de la elaboración de protocolos y guías clínicas que conduzcan a la calidad o excelencia del cuidado de enfermería, que es el centro de nuestro quehacer diario y cotidiano. Tal como lo señala el remanente teórico que "la práctica clínica basada en la evidencia, por lo tanto la enfermería basada en la evidencia debe originar que nuestras decisiones se basen en la utilización crítica y consciente de los resultados de investigación más reciente. Esto debe hacerse sin olvidar las preferencias y los valores de los pacientes, la experiencia personal y los recursos disponibles. Esta práctica se alimenta de un conjunto de herramientas modernas que se nutre del conocimiento que produce la epidemiología clínica" (1). Es decir, "que la utilización de la evidencia científica por parte de los profesionales de enfermería en la práctica asistencial, ha supuesto una mejora de calidad en los cuidados que realizan a los pacientes. Entendida la práctica basada en la evidencia como uno de los estándares más altos de calidad que ha demostrado mejorar los resultados en salud de los pacientes" (2).

El ejercicio basado en la evidencia como concepto general, fue abordado en un principio por los médicos, como enfoque dirigido a la solución de problemas surgidos en la práctica diaria. Por tanto, el quehacer diario de los distintos funcionarios es donde se va emplear la evidencia clínica (3). El conocimiento de la práctica de la enfermería parte con Florence Nightingale, como algo de sentido común, un arte propio e inherente al ser humano, pero de manera paulatina fue convirtiéndose en la profesión disciplinada que es en la realidad (3). En este sentido "la práctica de enfermería basada en la evidencia, independientemente de la controversia que genera la temática; hablar de evidencia científica en el cuidado de enfermería implica referirse a la producción científica en enfermería y de la aplicación de los resultados en el desempeño profesional. Es imposible desconocer la contribución que la práctica basada en evidencia ha realizado al auge actual de la investigación, y a su papel preponderante en el desarrollo y legitimización de las disciplinas" (4). Evidencia científica en enfermería es un instrumento para mejorar la práctica y el cuidado de las personas que atendemos a diario (3). Por tanto "a través de la enfermería basada en la evidencia (EBE) el profesional de enfermería puede acceder a un gran número de publicaciones científicas, evaluar su validez y aplicabilidad críticamente, lo más importante incorporarle a su práctica clínica cotidiana. Investigar en el campo de la enfermería es necesario como en cualquier disciplina y más cuando nuestro colectivo quiere demostrar que la enfermería es una profesión científica; permitiéndonos mejorar la calidad de la atención prestada a nuestros pacientes y así cumplir de forma satisfactoria con nuestro código deontológico y ético" (5). La enfermera, aplica el modelo científico a la práctica asistencial de sus 
cuidados a través del PAE (Proceso de Atención de Enfermería), permitiendo prestar cuidados de forma racional, sistemática, continua, realista y sobre todo basada en evidencias científicas (5). Puedo puntualizar como señala la literatura que "se debe incentivar una cultura de cambios en torno a la evidencia científica y transmitir esta información al grupo de trabajo; este debe acostumbrarse a compartir la utilidad de esta herramienta" (3).

Se concluye que es prioritario, trascendental e importante la enfermería basada en evidencia (EBE) porque es un instrumento que permite la utilización crítica, juiciosa de óptimos resultados de investigación científica, rescatar esta información, trabajar en equipo, elaborar protocolos y guías que conduzcan a la mejora de la calidad de los cuidados de enfermería.

Finalizo con este pensamiento: "Contribuye a la mejora de la calidad de los cuidados de enfermería, avance científico y tecnológico de nuestra profesión, aplicando el EBE" (Enfermería basada en evidencia).

\section{REFERENCIAS BIBLIOGRÁFICAS.}

1. Coello P, Esquerro O; Fargues I; García J, Marzo M, Navarra M, et al. enfermería basada en la evidencia. Hacia la excelencia en los cuidados. 1a ed. Madrid, España: Ed. DAE; 2004

2. Ansoain R. La evidencia científica en los cuidados de enfermería como mejora de calidad. Tesis para optar el Grado Master. Pamplona, España. Facultad de Ciencias de la Salud, Universidad Pública de Navarra; 2015.

3. Carrasco P. Importancia de la enfermería basada en evidencia en la elaboración de protocolos y guías clínicas. Santiago de Chile. 2004. Rev. Medware; 4(5)

4. Cañon H. la práctica de la enfermería basada en la evidencia. Bogotá, Colombia 2007. Rev. Investigar en enfermería: Imagen y derecho; 9(1): 101.

5. Domínguez S. Enfermería basada en la evidencia (EBE) Blog. Elsevier: España. 2013. 